\title{
Echter Emissionshandel statt heiteres Preise-Raten
}

\author{
Der Verkehrssektor gilt als Sorgenkind der deutschen Klimapolitik, \\ da hier bislang keine maßgeblichen Emissionsreduktionen erzielt \\ werden konnten. Im Klimapaket der Bundesregierung ist daher \\ vorgesehen, den Emissionshandel auf den Verkehr auszuweiten, \\ allerdings zunächst nicht als wirklichen Handel, sondern mit \\ Festpreisen. Ist dieser Ansatz zielführend? \\ Von Achim Lerch
}

$\mathrm{m}$ Klimapaket sind zunächst Festpreise von $10 €$ pro Tonne $\mathrm{CO}_{2}$, steigend auf $35 €$ vorgesehen. Dies geht anderen Akteuren, wie Teilen der Opposition oder den Umweltverbänden, nicht weit genug, gefordert werden Preise von 40,60 oder $80 €$ - bis hin zu mehr als $200 €$. Im Vermittlungsausschuss wurde schließlich als Kompromiss ein Einstiegspreis von $25 €$ vereinbart, steigend auf $55 €$. Der vorliegende Beitrag plädiert dafür, dieses Preise-Raten zu beenden und stattdessen einen echten Emissionshandel $\mathrm{zu}$ etablieren, mit Begrenzung der Emissionslizenzen und Preisfindung über den Markt.

\section{Die umweltökonomische Theorie}

Die Idee einer Umweltpolitik über Preise ist ja keineswegs neu. Jedoch scheitert eine Pigou-Steuer bekanntlich an der Schwierigkeit bis Unmöglichkeit, die externen Kosten exakt zu berechnen, weshalb in der heutigen Umweltsteuerdebatte der Standard-Preis-Ansatz von Baumol und Oates dominiert: Ein vorgegebener Emissionsstandard wird durch eine Abgabe erreicht, die den durchschnittlichen Grenzvermeidungskosten der Emittenten bei diesem Standard entspricht. Die Schwierigkeit dieses Ansatzes liegt im Problem der asymmetrischen Information: Während die Emittenten selbst ihre Grenzvermeidungskosten kennen, kann die Behörde diese allenfalls schätzen. Und das umweltpoli- tische Ziel wird bei dieser Preislösung nur erreicht, wenn die Schätzung richtig war. Anders beim Emissionshandel als Mengenlösung: Es werden nur Emissionsrechte in Höhe des gewünschten Emissionsstandards vergeben, die dann unter den Emittenten handelbar sind. Der Preis ergibt sich dann am Markt und spiegelt die tatsächlichen Grenzvermeidungskosten wider.

\section{Emissionshandel im Verkehr}

Dass auch im Verkehrsbereich niemand die tatsächlichen Grenzvermeidungskosten kennt, zeigt das heitere Preise-Raten, das derzeit die Diskussion beherrscht: Ob nun zehn oder $80 €$ pro Tonne $\mathrm{CO}_{2}$ die notwendigen Emissionsreduktionen bewirken, kann niemand wirklich seriös sagen. Deshalb wäre es sehr viel besser, einen wirklichen Emissionshandel $\mathrm{zu}$ etablieren, bei dem die Menge der Emissionsrechte auf den gewünschten Wert begrenzt ist und sich der Preis am Markt ergibt. Da dann die Erreichung des klimapolitischen Ziels gewährleistet wäre, bräuchte der Staat auch keine zusätzlichen (problematischen) Eingriffe in den Mobilitätsmarkt vorzunehmen, vielmehr könnten die Marktteilnehmer selbst technologieoffen über geeignete Maßnahmen zur Emissionsminderung entscheiden. Die dadurch eingesparten Kosten z. B. für die ökonomisch wie ökologisch fragwürdige staatliche Förderung der Elektromobilität (etwa die obendrein sozial unge- rechte Kaufprämie für Elektrofahrzeuge) könnten dann stattdessen genutzt werden, um mögliche soziale Härten abzufedern, die sich aus hohen $\mathrm{CO}_{2}$-Preisen ergeben könnten. Auch die Einnahmen etwa aus Versteigerungen der Emissionsrechte können für entsprechende Rückvergütungen an die Haushalte verwendet werden (Lerch 2011, Lerch et al. 2019). Preissteigerungen können ebenfalls begrenzt werden durch ein weiteres Vorantreiben der Verknüpfung des europäischen Emissionshandels mit anderen Systemen (Lerch 2017, Rudolph et al. 2017) und auch durch eine Verbindung des verpflichtenden mit dem freiwilligen Kohlenstoffmarkt: Eine stärkere Konzentration auf emissionsmindernde Kompensationsprojekte etwa in Afrika bietet nicht nur eine Reihe von Effizienzvorteilen, sondern schafft überhaupt erst die notwendigen Voraussetzungen, damit das in Afrika zu erwartende Bevölkerungswachstum klimapolitisch verträglich gestaltet werden kann (Radermacher 2019).

\section{Literatur}

Lerch, A. (2011): CO2-Emissionshandel - effizient oder gerecht? Zeitschrift für Sozialökonomie 48 (170/171), S. 39-47.

Lerch, A. (2017): Perspektiven der Klimapolitik nach Paris: Mehr Markt! UmweltWirtschaftsForum. Vol. 25, 2017, S. 265-267.

Radermacher, F. J. (2019): Zur Umsetzbarkeit der Agenda 2030 In: Herlyn, E. \& M. Lévy-Toedter (Hg.): Die Agenda 2030 als Magisches Vieleck der Nachhaltigkeit. Springer, S. 15-42.

Rudolph, S./Lerch, A./Kawakatsu, T. (2017): Emissionshandel von unten - Die Chancen einer nachhaltigen Verknüpfung regionaler Treibhausgas-Märkte in den USA und Kanada. Zeitschrift für Umweltpolitik und Umweltrecht 40 (2), S. 113-133.

Lerch, A./Rudolph, S./Kawakatsu, T. (2019): lustum Pretium Carbonei: $\mathrm{Wie} \mathrm{CO}_{2}$-Preise den Klimaschutz gerechter machen. ÖkologischesWirtschaften, Heft 3/2019, S. 10.

AUTOR + KONTAKT

Dr. Achim Lerch ist Professor für Volkswirtschaftslehre an der FOM Hochschule in Kassel.

FOM Hochschule, Hochschulzentrum Kassel, Kölnische Straße 69, 34117 Kassel. E-Mail: achim.lerch@fom.de 